\title{
Sonography of Partial-Thickness Quadriceps Tendon Tears With Surgical Correlation
}

Samuel La, MD, David P. Fessell, MD, John E. Femino, MD,

Jon A. Jacobson, MD, David Jamadar, MB, BS, Curtis Hayes, $M D$

\begin{abstract}
Objective. With the use of surgical findings as the reference standard, the purpose of this study was to describe the sonographic findings of partial-thickness and complete tears of the quadriceps tendon and to determine whether sonography can potentially aid diagnosis. Methods. Three hundred eightynine consecutive sonographic reports (January 1996 to April 2001) of the knee/quadriceps tendon were reviewed retrospectively and assessed for subsequent surgery on the quadriceps tendon. Seven cases were thus identified. Findings at surgery (complete versus partial tears) were compared with the original sonography reports. Results. All 4 partial tears and 1 of 2 complete tears were diagnosed correctly on the basis of sonography. One complete tear was described as a partial tear on the basis of sonography. In a seventh case, complete disruption of the extensor mechanism with osseous avulsion of the superior pole of the patella was identified correctly. Dynamic scanning was essential in diagnosing a partial quadriceps tendon tear in 1 case. Conclusions. Sonography, including the use of dynamic evaluation, was helpful in the diagnosis of partial-thickness tears of the quadriceps tendon and may aid in differentiation of such cases from complete quadriceps tendon tears, particularly in the acute setting. The presence of scar tissue in the setting of chronic injury may represent a potential pitfall in the assessment of partial versus complete quadriceps tears. Further study is needed to define the accuracy of sonography for detecting quadriceps tendon tears. Key words: knee; knee sonography; sonography; tendon injuries; tendons.
\end{abstract}

Received March 19, 2003, from the Departments of Radiology (S.L., D.P.F., J.A.J., D.J., C.H.) and Orthopedic Surgery (J.E.F.), University of Michigan Medical Center, Ann Arbor, Michigan USA; and William Beaumont Hospital, Royal Oak, Michigan USA (D.P.F.). Revision requested April 19, 2003. Revised manuscript accepted for publication May 20, 2003.

Address correspondence and reprint requests to David P. Fessell, MD, William Beaumont Hospital, 3601 W Thirteen Mile Rd, Royal Oak, MI 480736769 USA.

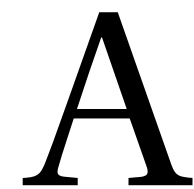

lthough uncommon when all types of injuries of the knee joint are considered, injury to the quadriceps tendon is the second most common injury to the extensor mechanism after patellar fracture. ${ }^{1}$ Quadriceps tendon injuries include a spectrum of disease ranging from tendinosis to partial-thickness tears to complete tendon rupture. In the older patient population, quadriceps tendon tears usually occur in the setting of a slip and fall. Usually there are preexisting degenerative changes within the tendon, thought to be due to degeneration or repetitive microtrauma. ${ }^{1,2}$ In younger patients, acute tears are usually sports related, due to sudden acceleration forces or extreme stress. Complete quadriceps tendon ruptures are treated surgically, whereas tendinosis is usually treated conservatively. 
Depending on the degree of the tear and the resulting loss of function, partial tears may be treated surgically. ${ }^{2}$ Accurate assessment of the degree of partial tears in conjunction with the degree of disability can aid the clinician in determining surgical versus conservative treatment.

Although many cases of quadriceps rupture can be diagnosed clinically, partial tendon tears may be more difficult to assess clinically because some degree of function is often maintained. When tears are acute, soft tissue swelling and associated hematoma may obscure the tendon defect on physical examination and may limit the clinical evaluation. ${ }^{2}$ With delayed presentation of a tear, the tendon defect may not be evident due to scar tissue formation. There may also be partial return of quadriceps function after several weeks, which can confuse the diagnosis. ${ }^{3}$ In 2 series, 13 of 53 quadriceps ruptures were diagnosed more than 2 weeks after injury, resulting in delayed repair.,4 Delay in diagnosis and treatment can result in considerable morbidity due to tendon retraction, fibrosis, and atrophy, which may necessitate tendon lengthening and more extensive surgery. ${ }^{2-4}$

Therefore, accurate imaging can aid diagnosis and can affect patient treatment directly. Radiographs are usually not diagnostic of quadriceps tendon tears. ${ }^{5}$ The use of magnetic resonance imaging for the diagnosis of quadriceps tendon tears has been advocated, but its accuracy has not been defined. ${ }^{6-8}$ Bianchi et $\mathrm{al}^{9}$ described the usefulness of sonography for complete quadriceps tendon tears with surgical correlation. With the use of surgical findings as the reference standard, the purpose of this study was to determine whether sonography could aid in the diagnosis of partial-thickness quadriceps tears and could differentiate them from complete tendon tears.

\section{Materials and Methods}

Institutional Review Board approval was obtained for this study. With the use of a database search, 389 consecutive sonographic reports (January 1996 to April 2001) of the knee/quadriceps tendon were reviewed retrospectively. All cases were assessed individually for subsequent surgery on the quadriceps tendon. Seven cases were thus identified. The study group included 5 men and 2 women (age range, 19-65 years; mean age, 44 years).
The original sonographic report was used, rather than retrospective review of the sonographic images, because the sonographer has more information than that contained in static images. Because tendinosis and partial tears often cannot be distinguished by imaging or clinical examination, surgical findings were chosen as the reference standard to provide the highest possible accuracy and strongest assessment of the sonographic findings. Radiographs obtained near the time of injury were also reviewed retrospectively by 1 musculoskeletal radiologist and compared with sonographic images.

The sonographic examinations were performed by 1 of 3 musculoskeletal radiologists with experience in musculoskeletal sonography. Scanning was performed with a $12-5-\mathrm{MHz}$ transducer (HDI 5000; Philips Medical Systems, Bothell, WA) with the patient in the sitting or supine position. Transverse and longitudinal sonograms were obtained. Scanning was performed with the knee at rest without application of traction on the patella. Dynamic scanning was performed as the patient flexed and extended the knee.

A complete tear was diagnosed when all 3 layers (1, rectus femoris; 2 , vastus medialis and lateralis; and 3 , vastus intermedius) of the quadriceps tendon were disrupted completely and the torn ends were separated by an anechoic or hypoechoic gap. A partial tear was diagnosed when a focal defect in fewer than all 3 layers was noted. ${ }^{8,9}$ Findings at surgery (complete versus partial tear) were compared with the original sonographic reports.

\section{Results}

In our study, patients fell into 3 general categories. One group comprised 5 adults (age range, 43-65 years; mean age, 53.4 years) with a mechanism of slip and fall. The second category comprised a 19-year-old patient who injured the tendon playing basketball. The third category comprised a 22-year-old patient with a mechanism of slip and fall but also with underlying osteoporosis. Review of the other patients' medical records did not reveal any predisposing underlying systemic disorders such as diabetes mellitus or gout. Injury to the quadriceps tendon was clinically suspected in all 7 patients before imaging studies. However, 2 had clinically sus- 
pected diagnoses more than 3 months after injury because of difficult clinical examination.

Surgical reports described 4 partial quadriceps tendon tears, 2 complete tears, and 1 osseous avulsion. Sonographic reports had correct diagnoses of all 4 partial tears, 1 of the 2 complete tears, and the osseous avulsion (Figs. 1-3).

Five of the 6 sonographic cases that provided correct diagnoses were evaluated in the acute setting. In this group, the sonographic examinations were performed 2 to 12 days from the dates of injury (mean, 6.8 days), and surgery was performed 7 to 40 days from the dates of injury (mean, 18.6 days). The sixth case was evaluated 3.5 months after injury and had complete disruption of the extensor mechanism with osseous avulsion of the superior pole of the patella. The quadriceps tendon was noted to attach to the fracture fragment with a tear of the underlying vastus intermedius tendon deep to the fracture.

One complete tear was described as a partial tear on the basis of sonography (Fig. 4). This patient underwent sonography 4 months from the time of injury and was taken to surgery 5 months after injury. Sonography in this case showed complete tears of the rectus femoris and vastus intermedius at the distal aspect of the tendon with a large separation between the ends of the tendon. Although the vastus medialis and lateralis did not have the normal fibrillar echo texture, linear echogenic structures were noted and thought to represent intact tendon fibers. At surgery, a complete tear was noted, with a large amount of scar tissue filling the tendon gap.

Dynamic scanning during flexion and extension of the knee was essential in making the diagnosis of partial quadriceps tendon tear in 1 case (Fig. 2). In extension, an apparent complete tear was noted. However, with flexion of the knee, intact deep fibers were visible. A partial quadriceps tendon tear was confirmed at surgery.

Six of 7 patients had radiographs of the knee (mean, 6.3 days after injury). These radiographs were retrospectively reviewed by 1 author. Five of 6 radiographs showed suprapatellar ossification or calcification. The 1 patient for whom osseous avulsion was prospectively described by sonography indeed had an ossific fragment superior to the patella at surgery. In 4 additional cases, 3 patients ( 2 with partial quadriceps tendon tears and 1 with a complete tear) had small avulsion

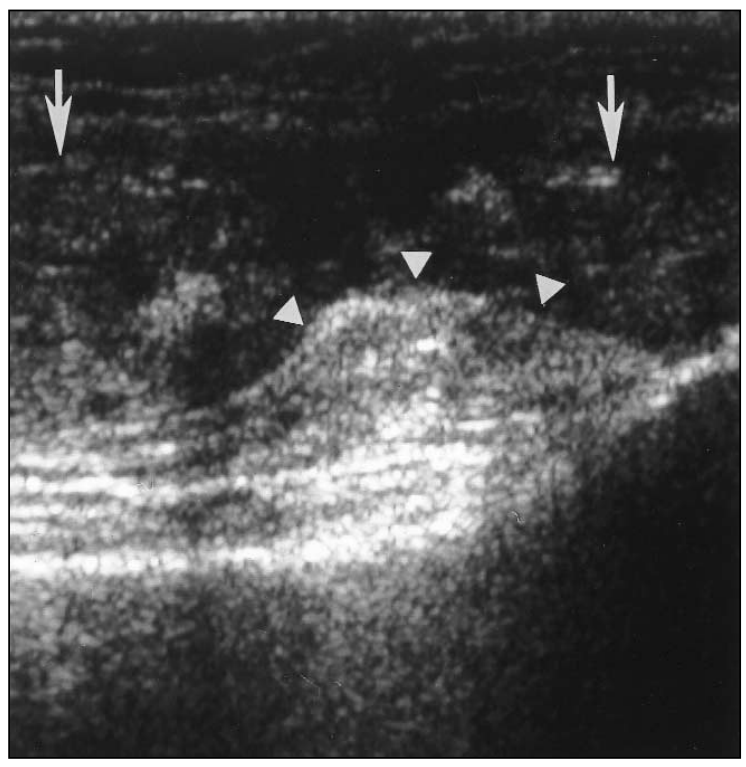

Figure 1. Full-thickness quadriceps tendon tear, shown on sonography and at surgery, in a 43-year-old man. The longitudinal sonographic image shows torn tendon ends (arrows) separated by a hypoechoic gap (between arrows). Echogenic prefemoral fat is herniating into the tendon gap (arrowheads). In all longitudinal images, cranial is left, and caudal is right.

bone fragments from the patella. In retrospect, these appeared as echogenic shadowing objects at the termination of the torn and retracted tendons on sonographic images. The remaining case (complete tear) had indeterminate calcification/ossification more proximally in the quadriceps muscle that was not imaged with sonography.

\section{Discussion}

The quadriceps group comprises 4 muscles: the vastus medialis, vastus intermedius, vastus lateralis, and rectus femoris. The rectus femoris originates from the anterior inferior iliac spine. The remaining quadriceps muscles originate from the femoral shaft. The quadriceps tendon represents the confluence of the 4 muscle tendons and inserts on the superior pole of the patella. The quadriceps tendon has 3 laminae: the most superficial lamina is formed by the rectus femoris; the middle lamina is formed by the vastus lateralis and vastus medialis; and the deep lamina is formed by the vastus intermedius. ${ }^{8}$ Partial tears may affect single or multiple layers, whereas full tears represent rupture of all 3 layers. 
To the best of our knowledge, only 1 prior sonographic study of quadriceps tendon tears was performed with surgical correlation. ${ }^{9}$ In that study, Bianchi et al $^{9}$ used sonography to diagnose 4 surgically confirmed complete tears and a single surgically confirmed partial tear. In our study of 7 patients, sonography facilitated correct diagnoses of all 4 surgically confirmed partial tears and 1 of 2 complete tears. In an additional case, complete disruption of the extensor mechanism with avulsion of the superior pole of the patella was identified correctly. With the higher number of surgically proved partial tears and the use of dynamic sonography, our study provides additional data to support the usefulness of sonography in diagnosing partial tears and distinguishing them from complete tendon tears.

When there is a delay between injury and sonographic evaluation, scar tissue can form in the tendon gap and may be confused with intact tendon fibers. This likely explains why 1 partial tear at sonography was noted to be a complete tear at surgery (Fig. 4). Hypoechoic or mixed echogenic scar tissue may have simulated intact tendon fibers. Alternatively, in the 1-month delay

Figure 2. Partial-thickness quadriceps tendon tear, shown on sonography and at surgery, in a 62-year-old woman, showing the value of dynamic assessment. A, Longitudinal sonogram at the level of the superior patella (arrowheads) performed with the knee in extension shows an apparent full tear (between arrows). B, Longitudinal sonogram with the knee in partial flexion reveals some intact tendon fibers (arrows) of the vastus intermedius. C, Transverse sonogram with the knee in partial flexion shows intact tendon fibers (arrows). Arrowheads outline the cortex of the distal femur.

B

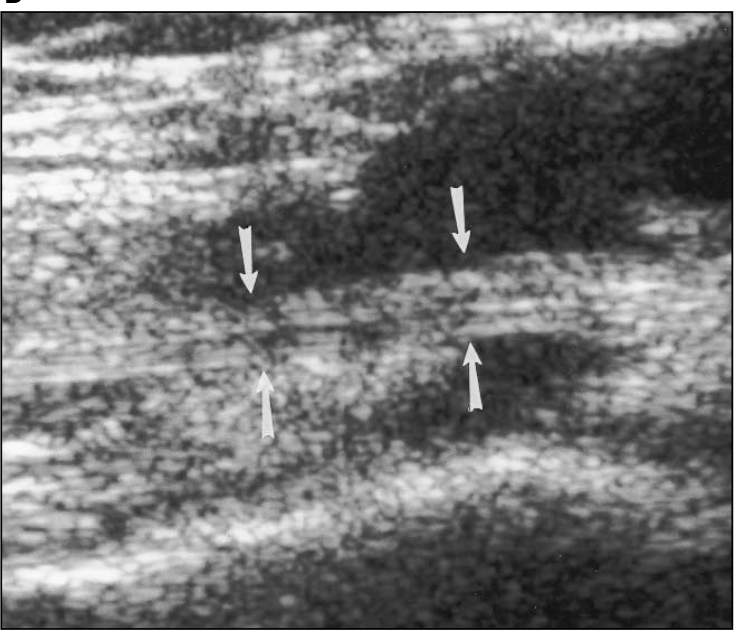

between sonography and surgery, a partial tear could have torn further, resulting in the complete tear found at surgery.

Real-time dynamic scanning is a feature unique to sonography and is a capability that should be used to optimize evaluation of tendon tears. With knee flexion and extension, the fibrillar echo texture of an intact tendon may be more obvious, allowing further differentiation from scar tissue. In addition, lack of tendon translation across an area of abnormality with joint movement or the appearance of a tendon gap or retraction supports the diagnosis of a complete tendon tear. In some cases, patient pain or discomfort may limit the ability to flex the knee. In such cases, gently applying traction on the patella may aid in the

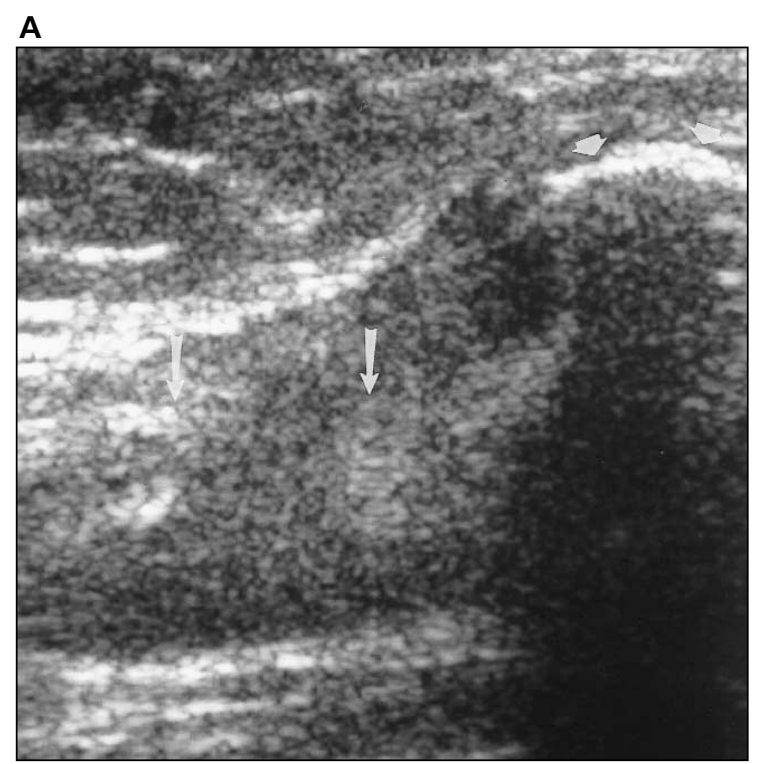

C

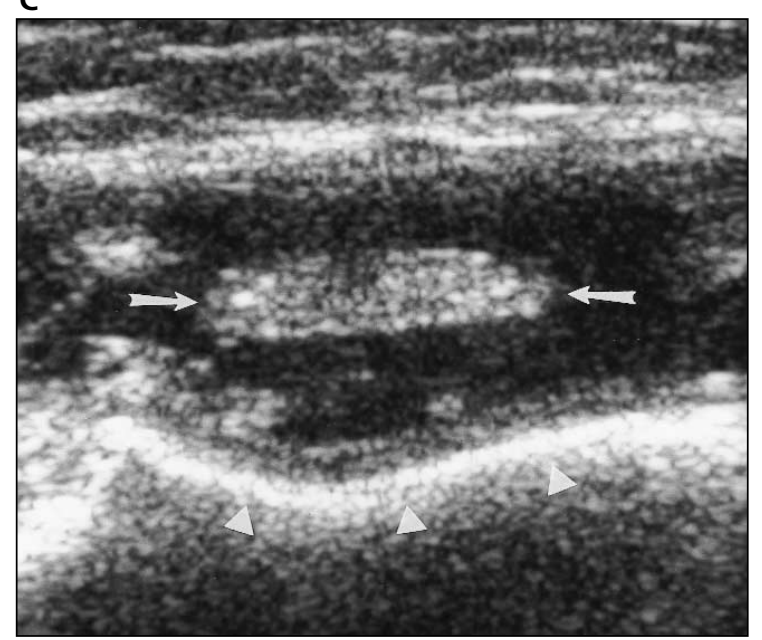


evaluation of tears by showing widening of the tendon gap in complete tears. The sonographer cannot be satisfied with noting a complete tear in a portion of the tendon. Complete scanning of the entire quadriceps tendon from the medial to lateral borders is essential for optimal diagnostic accuracy.

In the absence of a visualized tendon cleft, partial tendon tears and tendinosis often cannot be distinguished by imaging techniques or clinical evaluation. Both may coexist within a diseased tendon. ${ }^{10}$ Tendinosis is usually treated with conservative measures, whereas partial tears may be treated with surgery. ${ }^{2}$ Some imaging characteristics may help in differentiating tendinosis from a partial tendon rupture. In the setting of acute tendinosis, the tendon may appear swollen, in contrast to tendon thinning, which may be present at the site of a partial tendon tear. Tendinosis may not extend to the tendon surface, ${ }^{10}$ whereas a defect that extends to the tendon surface is indicative of a tear. A defined defect or gap in the tendon is consistent with a tear and was noted in all cases in this study, allowing discrimination from tendinosis.

Figure 3. Partial quadriceps tendon tear, shown on sonography and at surgery, and distal quadriceps tendon ossification, noted on radiography, in a 65-year-old man. A, Lateral radiograph of the knee shows ossification in the suprapatellar region. B, Longitudinal sonogram at the level of the distal quadriceps tendon shows ossification with posterior shadowing (small arrow) and no visible intact tendon. Hypoechoic debris fills the tendon gap (large arrows). Arrowheads mark the patella. C, Longitudinal sonogram with scanning more medially shows an intact quadriceps tendon (arrows) proximal to patellar insertion. Arrowheads mark the patella.

B

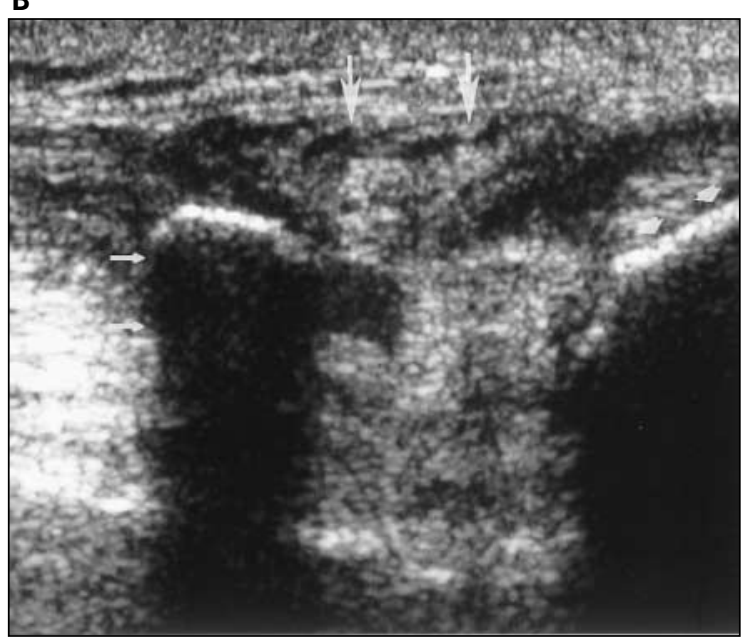

Calcification or ossification appears as an echogenic structure with posterior acoustic shadowing (Fig. 3). Calcification within the tendon is usually dystrophic, related to chronic injury. Such shadowing could potentially obscure underlying tendon pathology. Caution must be used when calcification is present. Scanning from a more medial or lateral approach may be useful in such cases and may allow visualization of structures underlying the calcification. Dynamic scanning also may aid evaluation. Similarly, caution must be used when there is osseous avulsion from the patella.

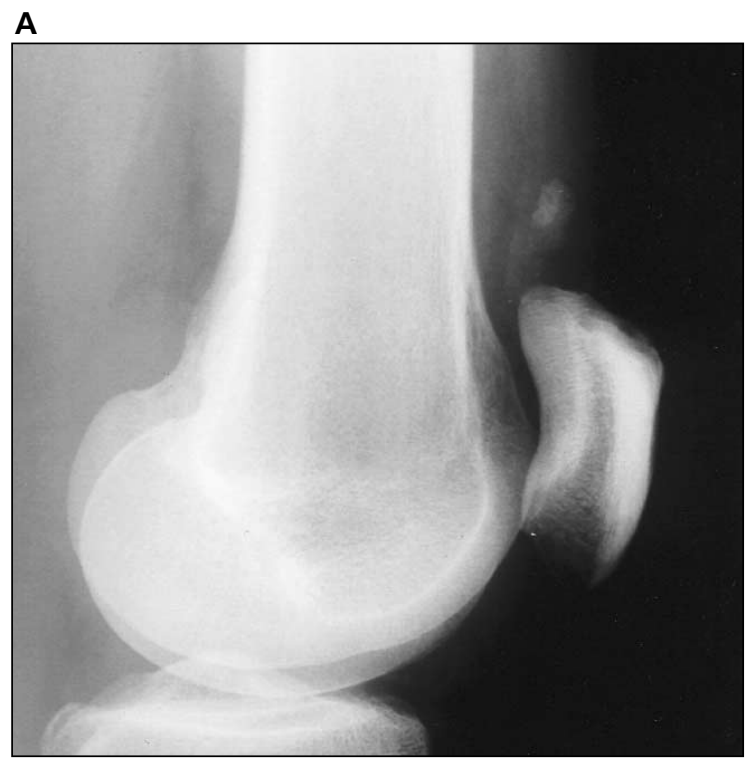

C

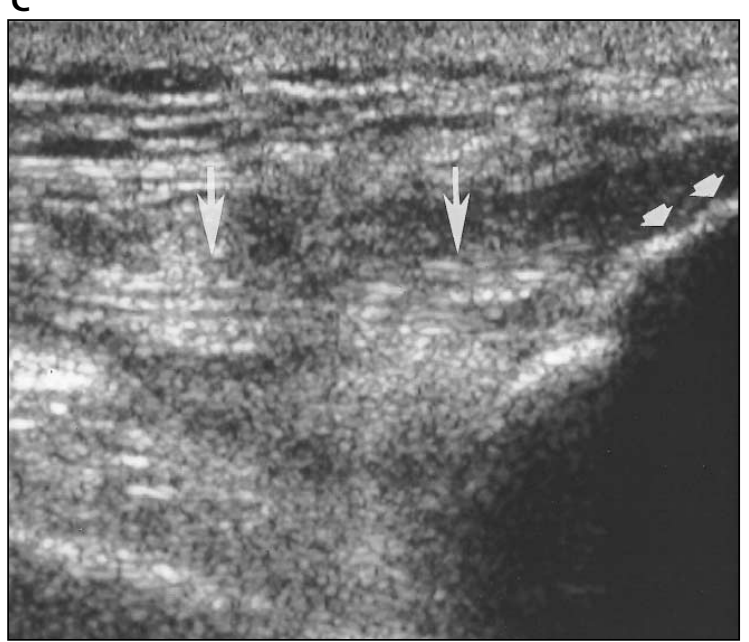


Identification of an echogenic and shadowing structure in the most distal aspect of a retracted tendon may indicate an avulsion fracture and can be a helpful finding. Interestingly, identification of ossification on radiography does not necessarily imply a complete rupture of the quadriceps tendon from the patella. Ossification representing osseous avulsion from the patella was present in 2 of our partial-thickness tendon tears (Fig. 3). These cases suggest that patellar avulsion may be seen in both partial and complete tendon tears. Although radiographic findings are frequently abnormal, they are often nonspecific, and sonography may provide additional valuable information in assessing the degree of tendon injury.

Figure 4. Full quadriceps tendon tear, shown at surgery with a partial tear diagnosed on the basis of sonography, in a 50-year-old man. A, Longitudinal sonogram shows torn tendon ends (arrows) and marked volume loss. B, Adjacent longitudinal sonogram shows linear echogenic structures thought to represent intact quadriceps tendon fibers (arrows). Fibrous/scar tissue was noted to fill the tendon gap at surgery. Sonography was performed 4 months after injury.

A

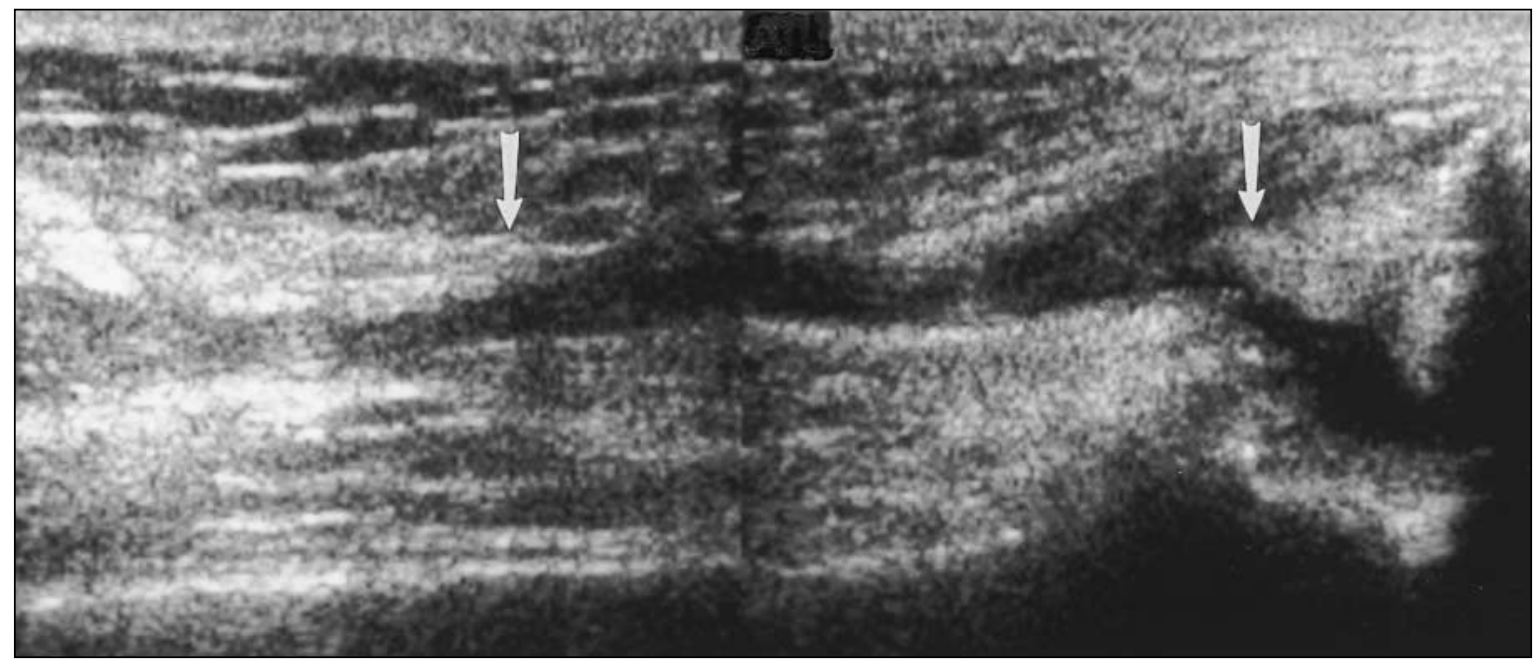

B

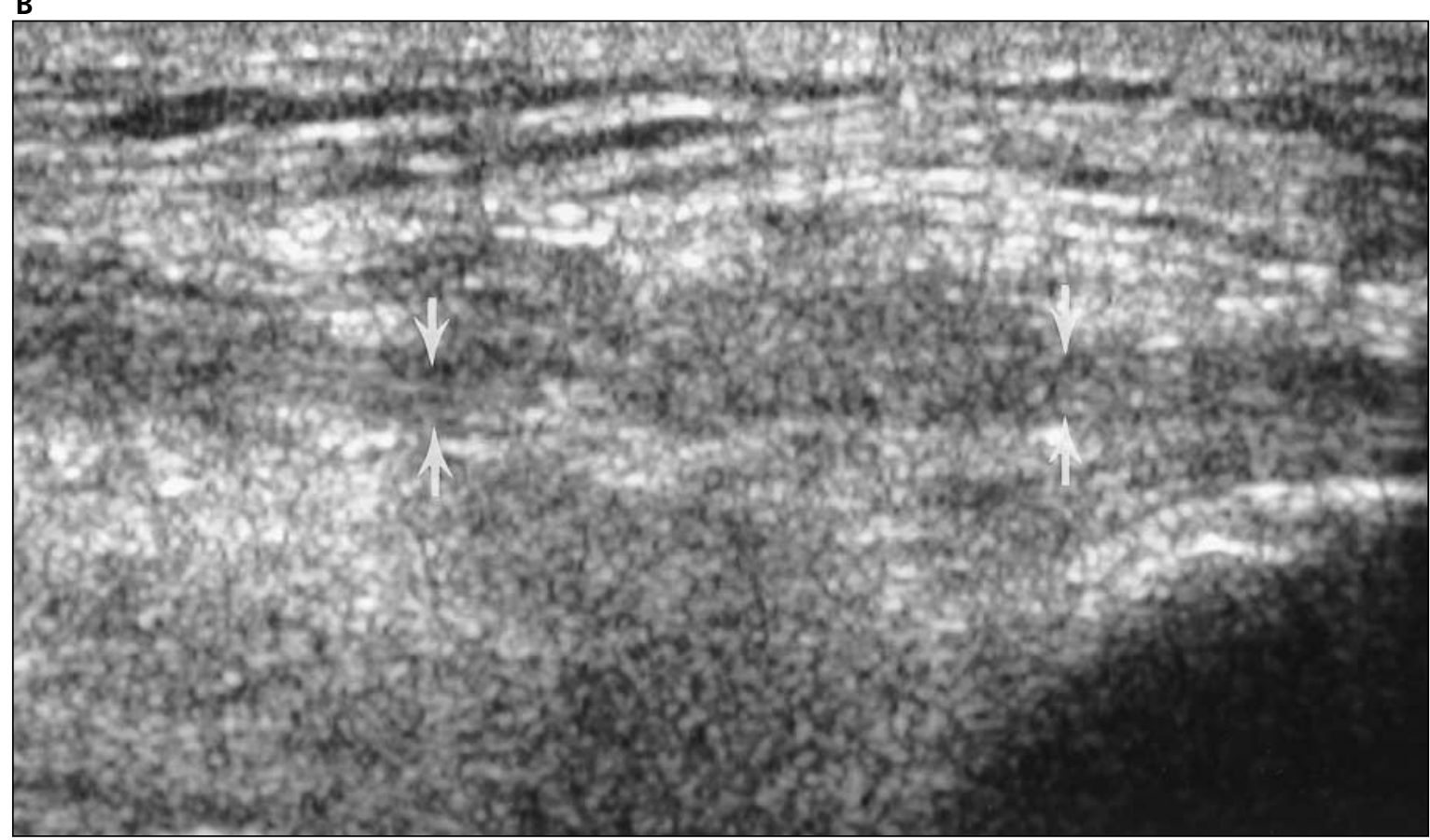


The study is biased by the inclusion of only surgically verified cases. Using surgical correlation as the reference standard prevents the inclusion of cases with no abnormalities, lowgrade tendinosis, and low-grade partial tears because these cases would not be treated surgically. However, surgical correlation provides the highest accuracy and is needed to establish the validity of the sonographic findings. The interval between sonography and surgery also may have allowed changes to occur, potentially introducing error. Given the small number of surgical cases, statistical accuracy for detecting partial tears and differentiating them from complete tears cannot be quantified meaningfully, and further study is needed.

In conclusion, given the relatively uncommon nature of this injury and only 1 known prior sonographic study of quadriceps tendon tears with surgical correlation, ${ }^{9}$ our study provides additional data to support the usefulness of sonography in the evaluation of partialthickness quadriceps tendon tears. Sonography, including dynamic evaluation, may be an effective tool in the evaluation of quadriceps tendon injury, particularly in the acute setting. The presence of scar tissue in the setting of chronic injury may represent a potential pitfall in the assessment of partial versus complete quadriceps tears. Further study is needed to determine the accuracy and to further define the clinical utility of sonography in the diagnosis of quadriceps injury.

\section{References}

1. Nance EP, Kaye JJ. Injuries of the quadriceps mechanism. Radiology 1982; 142:301-307.

2. Scuderi C. Ruptures of the quadriceps tendon. Am J Surg 1958; 95:626-635.

3. Ramsey RH, Muller GE. Quadriceps tendon rupture: a diagnostic trap. Clin Orthop 1970; 70:161-164.

4. Siwek CW, Rao JP. Ruptures of the extensor mechanism of the knee joint. J Bone Joint Surg Am 1981; 63:932-937.

5. Kaneko K, DeMouy EH, Brunet ME, Benzian J. Radiographic diagnosis of quadriceps tendon rupture: analysis of diagnostic failure. J Emerg Med $1994 ; 12: 225-229$.
6. Kuivila TE, Brems JJ. Diagnosis of acute rupture of the quadriceps tendon by magnetic resonance imaging: a case report. Clin Orthop 1991; 262: 236-241.

7. Yu JS, Petersilge C, Sartoris DJ, Pathria MN, Resnick $D$. MR imaging of injuries of the extensor mechanism of the knee. Radiographics 1994; 14:541-551.

8. Zeiss J, Saddemi SR, Ebraheim NA. MR imaging of the quadriceps tendon: normal layered configuration and its importance in cases of tendon rupture. AJR Am J Roentgenol 1992; 159:1031-1034.

9. Bianchi S, Zwass A, Abdelwahab IF, Banderali A. Diagnosis of tears of the quadriceps tendon of the knee: value of sonography. AJR Am J Roentgenol 1994; 162:1137-1140.

10. Jacobson JA. Musculoskeletal sonography and MRI: a role for both imaging methods. Radiol Clin North Am 1999; 37:713-735. 